\title{
A Hierarchical Model of Motor Skill for Haptic-assisted Virtual Reality Training
}

\author{
Dangxiao Wang, Yuru Zhang and Jun Wu
}

State Key Laboratory of Virtual Reality Technology and Systems, Beihang University, Beijing, 100191

\begin{abstract}
Model of motor skill pattern is a fundamental component to support feedback mechanism in haptic-assisted motor skill training. Because of the diversity of manipulation tasks in real world, it is a challenge to construct a generic model for various motor skill patterns. Considering fine motor skill mediated by a rigid tool, criteria for a general model of motor skill are identified: generality, quantifiable representation and the capability to support real-time error computation. A hierarchical motor skill model is proposed in a two-level architecture: the spatial-temporal coupling mechanism in task level and the quantified representation in action level. The purpose of task level is to decompose a motor skill into basic elements that can be described with quantified representation. The purpose of the action level is to give quantified representation in each single dimension in the Cartesian Space. Based on the combinations of independent variants, mapping functions in the action level are classified as five basic types. The proposed model can provide a systematical view to compare various motor skills in literatures, and thus can help us to identify unexplored topics in haptic-enabled motor skill training area. Furthermore, examples of two motor skill tasks show that the proposed model can provide a systematic way to design training system for complex motor skills.
\end{abstract}

Index Terms-Motor skill, hierarchical model, task level, action level, haptic training.

\section{INTRODUCTION}

\subsection{Motivation}

Motor skill training is an active field that aroused interest of researchers from cognitive science and psychophysics etc. [1-6]. Motor skill is usually defined as the capability of a subject to output motion and force. Almost all dexterous manipulations are dependent on "arrives appropriate position at exact time" or "output appropriate force at exact time”. For instance, injection and suture in surgery, bolt screwing in assembly and velocity control of welding torch etc. Recently, haptic assisted motor skill training is becoming an active topic [1]. In haptic human-machine systems, power transfer occurs between human and haptic device, which includes transfer of motion and force. Typical haptic-assisted training systems include surgical training, assembly training, and sports training etc.

Manuscript Received on 26 July, 2010

E-Mail: hapticwang@buaa.edu.cn
For motor skill training using haptic systems, the architecture can be summarized as shown in Fig. 1:

(1) Modeling: For a specified motor skill, establish the ideal motor skill pattern (or the pattern of expert subjects); and

(2) extract trainees' actual pattern through capture of real-time motion and/or force signal;

(3) Training: Compute the error between the ideal and actual pattern, and produce force signal based on some guidance rules. Sometimes combining with visual guidance signals, feedback cues are provided to the trainees during real-time practice process;

(4) Evaluation: Extract the performance data of multiple subjects before and after the training, and analyze the evolvement of motor skill.

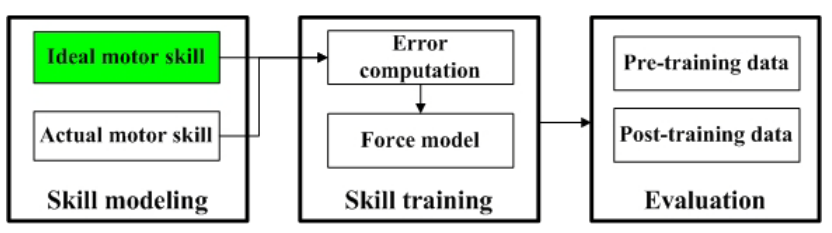

Fig. 1. Typical motor skill training architecture

From Fig. 1, error-based feedback is important in motor skill learning. In order to produce the error signal, it is necessary to describe the ideal motor pattern precisely (as shown in the green block in Fig. 1). Furthermore, the feedback should be clear and in real-time [1]. Therefore, the model of motor skill should be able to describe the difference between different tasks and should be able to describe the time related features.

In this paper, we focus on the modeling of motor skill (the gray block in Fig. 1) to study following problems:

(1) What is the similarity or difference among various types of motor skill tasks?

(2) How to quantify the motor skill and how to decompose a complex motor skill into a quantified model that can be executed by haptic controllers?

\subsection{Literatures review}

In literatures, descriptive definition of motor skill has been proposed. Singer et al. defined motor skill as the ability to execute a movement in an optimal fashion, or as an activity of a person involving a single or a group of movements performed with a high degree of precision and accuracy [2]. Gagne claimed that motor skill includes two aspects: the first is action program, and the second is actual muscle movement which can become more and more precise and continuous after practice with feedback [7]. 
In recent years, modeling and training method for specified manipulation tasks has been studied. Feygin et al. investigated the use of haptic information for learning a complex 3D trajectory. Three sinusoidal functions were integrated to form a complex 3D trajectory [8]. Srimathveeravalli et al. studied motor skill learning for handwriting. Two-dimensional trajectory function is used to describe the shape of characters [9]. Morris et al. studied force skill learning and they used sinusoidal force signal to model a single dimensional force skill [10]. Sewell et al. studied force skill training during egg drilling. The trainee is required to output a force bigger than a given threshold and maintain the force for a randomized period. When the resistance force vanishes suddenly, it is expected that the trainee can output minimized overshoot [11]. Lieberman et al. studied human's capability to control angle rotation precision under single joint and multiple-joint motion [1]. Edmunds et al. studied drilling skill against polystyrene and their goal is also to achieve minimized overshoot. Interaction process is divided into three statuses: non-contact, contact and penetration. Active event augmentation is utilized to provide a cue for the trainee to feel a sudden force change [12].

Summarizing previous works in literatures, there is no quantified representation for diverse motor skills. It is necessary to find a method to decompose complex motor skills into quantified components that can be executed by haptic controllers.

\subsection{Organization of this paper}

The remainder of this paper is organized as follows. In section 2, criteria for an ideal model and challenges are identified. In section 3, a hierarchical motor skill model and classification for mapping functions in the action level are proposed. In section 4, several motor skill task examples are discussed to validate the effect of the model. In section 5 , the potential of the model and future work are discussed.

\section{CHALLENGE FOR MOTOR SKILL MODELING}

\subsection{Requirement for motor skill model}

The principal function of a motor skill model is to translate a complex manipulation process into a command sequence that can be executed by haptic controllers. The sequence represents a goal of control of haptic device. Based on the requirement of motor skill training, specifications for a satisfied model of motor skill should meet three criteria: generality, quantify representation and the capability to support real-time error computation.

It should be noted that motor skill is a broad topic which involves multiple joints in human body. In this paper, we only consider fine motor skills mediated by a rigid tool which involve the movement of human's upper limb and hands.

\subsection{Feature analysis of modeling criteria}

Generality criterion means that the model is able to describe various motor tasks induced by the diversity of human's dexterous manipulation. As there are significant differences between different tasks, it is necessary to analyze features that induce to the diversity of motor skill tasks. The multiple-dimensional property of motor tasks is a main reason that leads to the difficulty of motor skill modeling. There are two ways for human to interact with environment: direct or indirect contact with environment. In direct contact, human's body part, such as hand or limb, contact objects. In indirect contact, a tool is used to manipulate objects. When human manipulate a tool against an object, he/she has to coordinate multiple joints to output expected motion or force. Table 1 gives typical features that describe the multiple-dimensional property of motor tasks.

TABLE 1: TYPICAL FEATURES OF MOTOR SKILL

\begin{tabular}{cc}
\hline Features & Possible choices \\
\hline Representation Space (RS) & Joint space/ Cartesian space \\
Involved Dimension (ID) & Single/ multiple \\
Dimension Coupling (DC) & Coordinative/ independent \\
Dimension Difference (DD) & Isotropy/ anisotropy \\
Environment Constraint (EC) & Free space/ constraint space \\
\hline Task Phase (TP) & Single/multiple \\
\hline
\end{tabular}

Representation space selection is a fundamental step for multiple-dimensional motor skill modeling. There are two typical choices: Joint space or Cartesian space. For the direct contact manipulation, it is convenient to describe motor skill in joint space. For the indirect contact manipulation mediated by a rigid tool, it is natural to describe motor skill in Cartesian space.

Involved dimension refers to numbers of spatial dimension necessary to describe the motion or force feature of the tool during manipulation. For manipulations mediated by a rigid tool, position/orientation and force/torque information of the manipulated tool is important instead of the rotation angle of operator's joints. Therefore, the involved dimension will vary from single dimension to multiple dimensions (six degree-of-freedom is maximum for a rigid tool).

Dimension coupling refers to coupling feature among all the involved dimensions. There are two possibilities: independent or coordinative.

Dimension difference refers to skill pattern type on different dimensions. There are two possibilities: isotropy or anisotropy. Isotropy means the pattern type on each dimension is the same while anisotropy means different pattern types are required for different dimensions.

Environment constraint reflects different force/movement requirements in motor skill modeling. There are two typical types of motor skills, the first is manipulation in free space, i.e. the tool does not contact with the environment. Typical example is trajectory of baton produced by a conductor in an orchestra. The second type is manipulation in constraint space. Typical example is drilling operation against bone by a surgeon.

This paper focuses on manipulation tasks mediated by a rigid tool and there exists contact between the tool and the environment. Therefore, the involved dimension is six degree of freedom, and the Cartesian space is used to describe the skill while the detailed movement of different joints of human body 
is not considered.

In order to get a quantify representation for the motor skill, it is necessary to provide quantified and efficient description for a motor sequence. In order to get the error between an expert and a trainee, it is a common method to record the manipulation data of expert using motion capture method. It is necessary to provide a model to organize or fit these data in order to compute error in real-time training. A popular method is to compare these two data series, e.g. dynamic programming [10] or computation of minimal distance for virtual fixture method $[13,14]$. In order to meet these requirements, it is necessary to organize these data into a high-efficient representation.

\section{A HIERARCHICAL MODEL OF MOTOR SKILL}

\subsection{A hierarchical model for motor skill}

In order to meet the three criteria, a hierarchical architecture for motor skill model is proposed, which include task and action level as shown in fig. 2 Motor skill such as suturing or knot tying skill is a complex process composed of several tasks [15]. The purpose of task level is to decompose it into basic elements that can be described with quantified representation. The purpose of the action level model is to give quantified representation in one single dimension in the Cartesian Space.

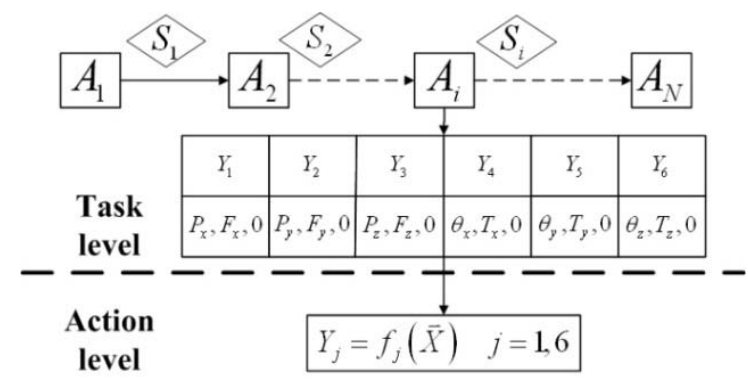

Fig. 2. Sequence of Action units and switch conditions.

\subsubsection{Task-level}

The task level model serves three functions: the spatial relationship (SR) among single dimension, the temporal relationship (TR) among single dimension and the switch condition (SC) between different motor skill elements. For example, a typical surgery includes several steps such as knot tying, suturing, stitching etc. Every step can be further divided into several sub-tasks. It is firstly important to identify involved dimensions for each sub-task.

As shown in Fig. 2, several actions form a time sequence of a complex motor skill. $A_{i}$ refers to a specified action unit in the skill. $S_{i}$ refers to switch condition between successive action units. $N$ refers to the number of action units in the skill.

In Fig. 2, $Y_{j}$ refers to the target vector component (controlled goal) of the specified action unit. As described before, it is enough to use six degree of freedom to describe motion and force statuses of a rigid tool. Because the tool's position and the force applied by the operator are coupled by the environment, it cannot be the control goal in same dimension simultaneously. And

$$
\vec{X}=\{\vec{F}, \vec{P}, t\}
$$

refers to the independent variables group of the action unit. $\vec{P}$ means the position and the orientation of the tool's proxy (i.e. the haptic interface point). $\vec{F}$ means the force and the torque imposed upon the tool's proxy. $t$ means the time sample.

$$
\left\{\begin{array}{l}
\vec{P}=\left[P_{x}, P_{y}, P_{z}, \theta_{x}, \theta_{y}, \theta_{z}\right] \\
\vec{F}=\left[F_{x}, F_{y}, F_{z}, \tau_{x}, \tau_{y}, \tau_{z}\right]
\end{array}\right.
$$

\subsubsection{Action-level}

In the task level, SR describes coupled relationship between action elements from single dimension to form a six-dimensional task. TR describes the time sequence among different elements, and also the idle time period between successive elements. For example, in Chinese character handwriting, there are multiple strokes in one character. Action of writing two successive strokes is an example of time sequence. SC describes switch condition that distinguishes two elements. For example, in drill task, there is a sudden force change or acceleration when the breakthrough happens [12]. Thus, breakthrough can be chosen as a switch condition.

In order to get quantified model for motor skill, the above six dimensional mapping is further decomposed into action level model in single dimension or in combined dimensions.

$$
Y_{i}=f_{i}(\vec{X})
$$

where $f_{i}(\square)$ refers to the function from independent variable $\vec{X}$ to target variable $Y_{i}$.

The features of the action element in action level can be summarized as: quantified representation, single or combined dimension for the target variable, and easy to program by digital computation.

\subsection{Classification of mapping function in action level}

The function of the action level model in equation (3) is to give a mapping function from independent variable $\vec{X}$ to target variable $Y_{i}$. Analyzing all the possible combination of target variable and independent variables during tool-environment manipulation process, classification of action element in action level can be summarized in table 2. Because displacement vector, force vector and time are three basic elements in tool-environment manipulation process, the combination in table 2 can cover all possible tasks in single dimension. It should be noted that, in Spatial-type and Muscular-type, independent variables are always located in different dimension from that of target variable.

\subsubsection{Spatial-type}

Spatial-type motor element describes the moving trajectory pattern of tool in Cartesian space. This type of skill is independent of time. Typical example includes Chinese handwriting using a pen. Only the trajectory of pen's tip is 
required to determine a specified shape instead of the velocity and the force against the paper. Another example is shape drawing in rehabilitation after strokes [4]. The patients are usually required to draw a circle and the shape is the only criteria to evaluate the task performance.

TABLE 2: CLASSIFICATION OF ACTION ELEMENT IN ACTION LEVEL

\begin{tabular}{|c|c|c|c|}
\hline \multirow{2}{*}{$Y_{i}$} & \multicolumn{3}{|c|}{$\vec{X}$} \\
\cline { 2 - 4 } & $\vec{P}$ & $\vec{F}$ & $t$ \\
\hline$Y_{i} \in \vec{P}$ & Spatial-type & $\begin{array}{c}\text { Hybrid-type } \\
\text { (admittance) }\end{array}$ & Spatial-temporal-type \\
\hline$Y_{i} \in \vec{F}$ & $\begin{array}{c}\text { Hybrid-type } \\
\text { (impedance) }\end{array}$ & $\begin{array}{c}\text { Muscular-type } \\
\text { (time } \\
\text { independent) }\end{array}$ & $\begin{array}{c}\text { Muscular- temporal - } \\
\text { type } \\
\text { (time dependent) }\end{array}$ \\
\hline
\end{tabular}

\subsubsection{Spatial-temporal-type}

Spatial- temporal -type motor element is an extended type of spatial type motor tasks enhanced by temporal characteristics or constraints. Usually, one order and two order derivative of trajectory on time is denoted as Velocity pattern and Acceleration pattern motor element.

A typical example of velocity pattern is control of welding torch. It is required that the torch should not only move exactly along the welding line (trajectory control), and but also maintain near constant velocity (velocity control). A typical example of Acceleration pattern is the drilling operation in dental surgery. Just at the moment when the drill breaks through dentin into dental pulp, the dentist should stop the movement of drill and should maintain minimal overshoot of the drill.

Velocity pattern can be classified into two kinds: velocity vs. time and velocity vs. position pattern, i.e. $\dot{\vec{P}}=f(t)$, $\dot{\vec{P}}=f(\vec{P})$. The second one means the tool is required to arrive at specified point at specified velocity.

\subsubsection{Muscular- type}

Muscular- type motor element describes the force skill when we only care about force/torque instead of its temporal or spatial characteristics. Typical example is the egg drilling task [11]. The operator needs to exert a force bigger than a threshold and then the egg can be drilled, and there is no exact requirement of the force-time or force-position pattern in this skill.

Another example is to train the force-maintaining-capability of patients in rehabilitation, i.e. to first train human to output a constant force, that is, to maintain precise force control capability.

\subsubsection{Muscular- temporal - type}

Muscular- temporal - type motor skill is dominated by force/torque pattern, and the pattern is time dependent. In Muscular- temporal - type, the model is:

$$
\vec{F}=f(t)
$$

\subsubsection{Hybrid-type}

Hybrid-type pattern is dominated by impedance/admittance pattern, and the pattern is time independent or time dependent. Impedance pattern can be modeled as:

$$
\vec{F}=f(\vec{P}, \dot{\vec{P}}, \ddot{\vec{P}})
$$

Admittance pattern can be modeled as:

$$
\vec{P}=f(\vec{F})
$$

\subsection{Mathematical representation of mapping functions}

Mathematical representation of mapping functions in action level is shown in Fig. 3. A discrete task is defined by a distinct beginning and endpoint, such as catching a ball. For continuous task, there are two types of representation forms: analytic function and numeric function.

In analytic function, the model can be derived as implicit or parametric functions:

$$
f\left(P_{x}, P_{y}, P_{z}, \theta_{x}, \theta_{y}, \theta_{z}\right)=0
$$

where the function could be linear or non-linear functions.

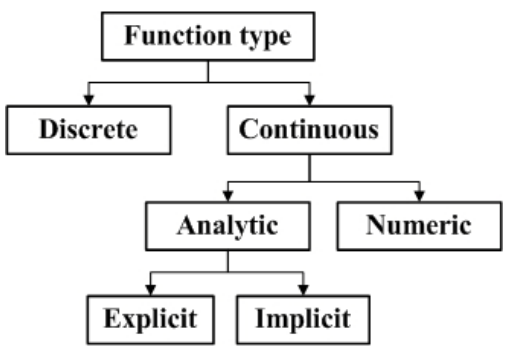

Fig. 3. Mathematical representation of mapping functions

Numeric function is necessary to describe complex trajectory that cannot be modeled by analytic function. Typical model include: line segment combination, piece-wise Bezier curves and their combination. As shown in Fig. 4, strokes of Chinese character are modeled by combination of line segments and piece-wise Bezier curves [16]. This method can be extended into motor tasks with higher dimensions, i.e. both position and orientation constraints. Typical example is tool's trajectory during suturing operation shown in Fig. 5 [18].

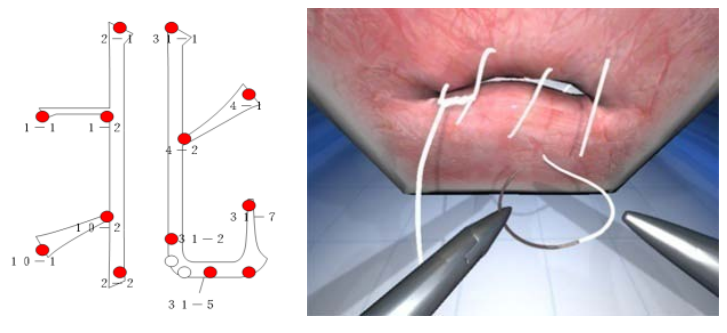

Fig. 4. Modeling of Chinese strokes Fig. 5. Suturing task

\section{V ALIDATION OF MOTOR SKILL MODEL}

What benefits can the hierarchical model provide for motor skill learning? The authors think it can help in two aspects:

1) to provide a systematic view to compare various motor skills in literatures, and thus can help us to identify some unexplored topics in haptic-enabled motor skill training area. This benefit will be explained in section 4.1 .

2) to provide a systematic way to design training system for complex motor skills. This benefit will be illustrated by modeling of two tasks in section 4.2 and section 4.3.

\subsection{Taxonomy of related works using proposed model}

In order to verify the generic metrics, related works in motor skill training is summarized using the proposed classification 
TABLE 3: CLASSIFICATION OF RELATED WORKS IN TWO-LEVEL ARCHITECTURE

\begin{tabular}{|c|c|c|c|c|c|c|}
\hline Task Features & Handwriting $* 1$ & Force skill [10] & Guidance [8] & Egg drill [11] & Joint angle [1] & Drill [12] \\
\hline \multicolumn{7}{|c|}{ Task level } \\
\hline $\mathrm{RS}$ & Cartesian space & Cartesian space & Cartesian space & Cartesian space & Joint space & Cartesian space \\
\hline ID & 3 & 1 & 3 & 1 & $1+$ & 3 \\
\hline $\mathrm{DC}$ & coordinative & 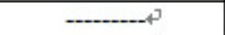 & Independent & ים & 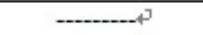 & coordinative \\
\hline $\mathrm{DD}$ & anisotropy & קיגוברבוב-ב & isotropy & קים & 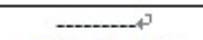 & anisotropy \\
\hline $\mathrm{EC}$ & constraint space & constraint space & free space & constraint space & free space & constraint space \\
\hline $\mathrm{TP}$ & multiple & single & single & 2 & single & 2 \\
\hline \multicolumn{7}{|c|}{ Action Level } \\
\hline Element type & Spatial-type & $\begin{array}{c}\text { Muscular- } \\
\text { temporal - type }\end{array}$ & $\begin{array}{c}\text { Spatial- temporal- } \\
\text { type }\end{array}$ & Muscular-type & Spatial-type & $\begin{array}{l}\text { Hybrid-type } \\
\text { (impedance) }\end{array}$ \\
\hline
\end{tabular}

* Task notes: Handwriting*1 refers to work in ref $[9,16,17]$.

method, which validate whether the proposed model is able to describe the diversity of various motor skill tasks.

Classification of related works using the proposed two-level architecture is given in table 3 . From this table, we can find that the proposed architecture can provide a systematical view to compare various motor skills in literatures, and thus can help us to find some unexplored topics in haptic-enabled motor skill training area, such as: (1) Velocity and acceleration pattern in Spatial-temporal-type skill; (2) Hybrid-type (admittance pattern) skill; (3) Multiple-dimensional tasks: the number of dimension is bigger than three dimensions.

Furthermore, we can find that existing work are mainly targeting simple motor skill tasks, which are only a small group of complex motor tasks in daily life. To author's knowledge, few works discuss how to design training system for more complex motor skills. The proposed architecture can provide a two-level systematic procedure to design training system for complex motor skills.

\subsection{Task 1: Dental drilling}

The proposed architecture can provide a two-level systematic procedure to design training system for complex motor skills. In order to verify the quantification metrics, model for dental drilling skill are explained. Especially, the spatial-temporal coupling mechanism in task level and the mathematical function in action level are formulated.

Fig. 6 shows scenario of dental drilling. The task requires a dentist to drill a hole along a straight line, passing three tissues: gum, dentin and pulp [18]. Just at the moment when the drill breaks through dentin into dental pulp, the dentist should stop the movement of drill and should maintain minimal overshoot of the drill. Because the dentist cannot observe the depth of drill inside the tooth, therefore, force feedback is indispensable to achieve high-quality drill result.
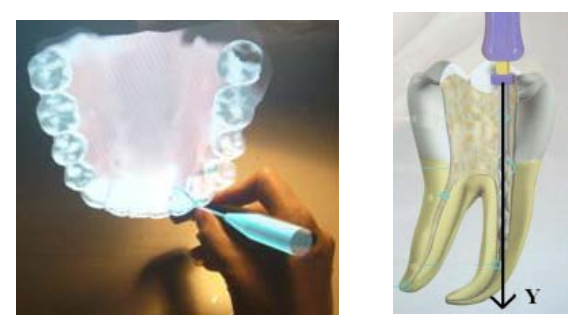

Fig. 6. Scenario of dental drilling
Suppose the feed forward direction of the drill is axis Y. The initial point where the drilling begins is $Y=0$, and the drilling is going toward positive axis. It is required to drill specified length. Base on the proposed motor skill model, the dental drilling skill can be described in Fig. 7. The dental drilling includes two phases. In the first phase, there are three dimensions involved. In the second phase, there is only one dimension involved.

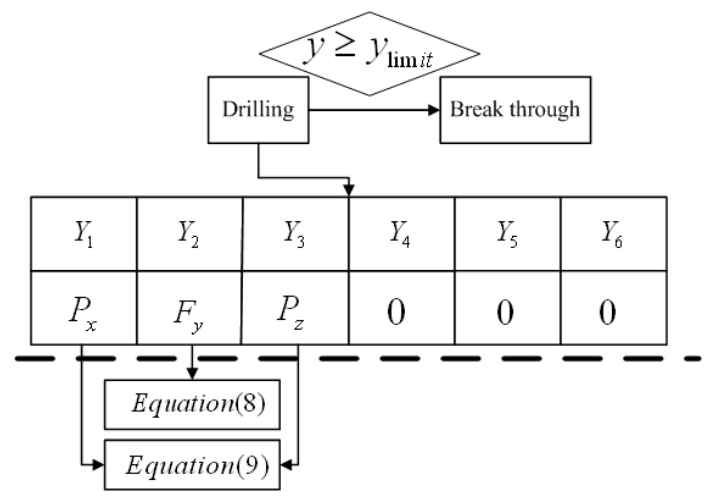

Fig. 7. Hierarchical models for dental drilling task

Quantified model for action level can be derived. In the first phase (drilling phase), the task belongs to anisotropy type skill. Along axis Y dimension, the task belongs to Muscular-type skill element. Furthermore, the function of force signal is discrete type, i.e. the force only needs to locate within a specified range as shown in equation (8). The force should be bigger than a force threshold $F_{\text {Low }}$, otherwise the material will not be removed; on the other side, the force should be smaller than a specified value $F_{\text {High }}$, otherwise the drill will be destroyed. In equation (8), $y_{\text {limit }}$ refers to the ideal depth of the hole.

When the specified depth of drilling hole is achieved or the breakthrough occurs, the active force needs to be stopped, that means the second phase of the skill element as shown in equation (9). The overshoot should be maintained as a minimized value, which means the human subject needs to control his acceleration when the drill breaks from dentin to the dental pulp. He needs to retreat his active force when the breakthrough occurs.

Within the perpendicular surface of the feed forward axis 
(Axis Y), the skill element belongs to Spatial-type element. The ideal goal is to maintain the drill along the feed forward axis. The trajectory control law can be described as shown in equation (9). Where $x^{*}, z^{*}$ refers to the ideal position of drill within the perpendicular surface (XOZ plane).

$$
\begin{array}{lr}
F_{\text {High }}>F_{y}>F_{\text {Low }} & 0<y<y_{\text {limit }} \\
X=x^{*}, z=z^{*} & 0<y<y_{\text {limit }}
\end{array}
$$

In the second phase, force pattern skill is derived as follows

$$
F_{y}=0 \quad y \geq y_{\text {limit }}
$$

\subsection{Task 2: Chinese brush-pen handwriting}

In order to verify the real-time computation metrics, the time-cost results for handwriting motor skill tasks are given to show the model is capable to support high update rate collision detection about $1 \mathrm{KHz}$.

For Chinese brush-pen handwriting, handwriting within each stroke can be defined as an action unit. The movement from the end-point of current stroke to the start point of next stroke can be defined as another kind of action unit, which is defined as idle action between successive strokes. In Fig. 8, the idle unit belongs to discrete-type action, which means a point-to-point manipulation. In the stroke writing unit, force control is needed in the direction normal to the paper plane, and trajectory control is needed in the paper plane. Suppose the normal direction is along axis Z. As shown in Fig. 9, the width of the stroke will increase according to the increase of normal force. For the handwriting of expert, the width of each stroke can be controlled nearly consistent, and also the shape of each character is correct.

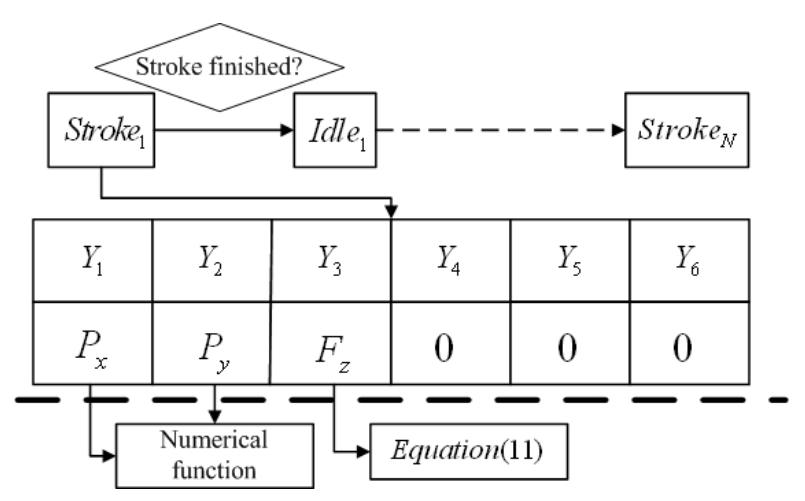

Fig. 8. Hierarchical models for handwriting task
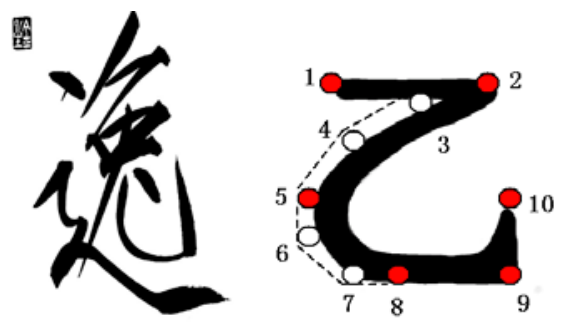

Fig. 9. Width change in character Fig. 10. Complex stroke
The model of action level for each stroke is:

- Along axis Z, the task belongs to Muscular-type skill. The magnitude of force is nearly constant. The model is shown in equation (11). $F_{z}^{*}$ is ideal normal force. $\delta$ is allowed fluctuation;

- Within the paper plane, the element belongs to Spatial-type. Because the diversity of Chinese character shape, it is impossible to use analytic function to model the trajectory. Hybrid model combined line segment and piece-wise Bezier curve is proposed to model the ideal trajectory [16]. Typical complex stroke for Chinese character is shown in Fig . 10. The number means the index of control points that define the ideal sequence of the pen movement.

$$
\left|F_{z}-F_{z}^{*}\right|<\delta
$$

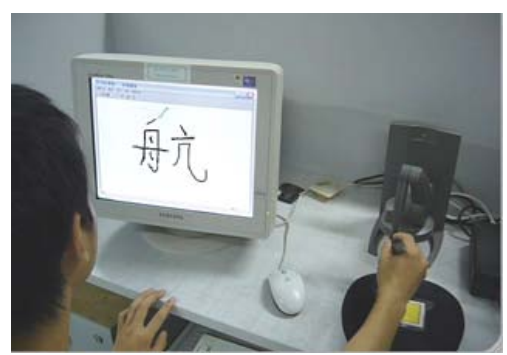

Fig. 11. A physical prototypes for Chinese brush-pen handwriting

A physical prototype based on Phantom desktop is established as shown in Fig. 11. Computation time for collision detection within the paper plane can be maintained less than $1 \mathrm{~ms}$ for different types of strokes including complex strokes [16].

\section{CONCLUSION AND DISCUSSION}

A two-level hierarchical model for motor skills is proposed in this paper, which includes the spatial-temporal coupling mechanism in task level and the quantified representation in action level.

In task level, skill features are described by several actions to form a time sequence of a complex motor skill. Skill elements are organized by three components: the spatial relationship among single dimension, the temporal relationship among single dimension and the switch condition between different motor skill elements.

In action level, the elements are classified into five types: spatial type, spatial-temporal type, muscular type, muscular-temporal type and hybrid type.

Based on the analysis of previous works, it is illustrated that the proposed model provides a systematic view to compare various motor skills in literatures, and thus can help us to find unexplored topics in haptic-enabled motor skill training area.

Using the dental drilling and the Chinese handwriting as examples, we can conclude that the proposed model is capable to decompose complex motor skill tasks into a quantified model that can be executed by haptic controllers, and thus to 
provide a systematic way to design training system for complex motor skills.

Future work includes real-time computation of error signal, which should be computed based on the ideal skill pattern and actual manipulation data of trainee. Furthermore, selection of suitable force model for different kinds of motor skills needs to be studied.

\section{ACKNOWLEDGEMENT}

This work is supported by the National Science Foundation of China under grant No: 60605027, and by National Hi-tech Research and Development Program of China under grant No: 2007AA01Z310. Their support is greatly appreciated.

\section{REFERENCES}

[1] Jeff Lieberman, Cynthia Breazeal, TIKL: Development of a Wearable Vibrotactile Feedback Suit for Improved Human Motor Learning, IEEE Trans. on Robotics, 23( 5): OCTOBER 2007 , pp.919-926

[2] R. N. Singer, "Motor Learning and Human Performance: An Application to Motor Skills and Movement Behavior" , Macmillan Publications, 3rd Edition, 1980

[3] M.K. O'Malley, A. Gupta, M. Gen, Y. Li. Shared Control in Haptic Systems for Performance Enhancement and Training. J Dynamic Systems, Measurement, and Control, 128.1, pp.75-85, March 2006

[4] J. Patton, F. A. Mussa-Ivaldi. Robot-Assisted Adaptive Training: Custom Force Fields for Teaching Movement Patterns. IEEE Trans BioMed Engr 51.4, 2004

[5] E. Bilodeau and I. Bilodeau, "Motor-skills learning," Annu. Rev. Psychol., vol. 12, pp. 243-280, 1961.

[6] P. M. Fitts, The Information Capacity of the Human Motor System in Controlling the Amplitude of Movement. Journal of Experimental Psychology, Vol. 47 (1954) 381-391

[7] J. Solis, C.A. Avizzano, M. Bergamasco, Validating a Skill Transfer System Based on Reactive Robots Technology, Proceedings of the 2003 IEEE international Workshop on Robot and Human Interactive Communication, Millbrae. California. USA, Oct. 31 -Nov. 2, 2003

[8] D. Feygin, M. Keehner, F. Tendick. Haptic guidance. Experimental evaluation of a haptic training method for a perceptual motor skill. In Proc. of the IEEE Virtual Reality Conference (HAPTICS), (2002)

[9] G. Srimathveeravalli, K. Thenkurussi, Motor skill training assistance using haptic attributes, Proceeding of 1st World Haptics conference, Mar. 18-20, 2005, Pisa, Italy

[10] D. Morris, H.Z. Tan, F. Barbagli, T. Chang, and K. Salisbury, "Haptic Feedback Enhances Force Skill Learning”, in Proceedings of the 2007 World Haptics Conference, Tsukuba, Japan, Mar. 22-24, 2007. pp. 21 26

[11] Christopher Sewell, Nikolas H. Blevins. Sumanth Peddamatham, Hong Z. Tan, Dan Morris. Kenneth Salisbury, The Effect of Virtual Haptic Training on Real Surgical Drilling Proficiency, in Proceedings of the 2007 World Haptics Conference, Tsukuba, Japan, Mar. 22-24, 2007. pp. 601-603

[12] Timothy Edmunds, Dinesh K. Pai, Perceptual Rendering for Learning Haptic Skills, Proceedings of the Symposium on Haptic Interfaces for Virtual Environments and Teleoperator Systems, 2008 Haptics Symposium, Reno, Nevada, U.S. Mar. 13 14, 2008

[13] G. Srimathveeravalli, V. Gourishankar, T. Kesavadas, "Comparative Study: Virtual Fixtures and Shared Control for Rehabilitation of Fine Motor Skills," Proceedings of the 2nd world haptics conference(2007) pp. $304-309$

[14] L.B. Rosenberg, "Virtual fixtures: Perceptual tools for telerobotic manipulation”, Proceedings of Virtual Reality Annual International Symposium (1993). pp.76- 82

[15] Richard Paul Holbrey, Virtual Suturing for Training in Vascular Surgery, Submitted in accordance with the requirements for the degree of Doctor of Philosophy, The University of Leeds, November 2004
[16] D. Wang, et al., Strokes-based modeling and haptic skill display for Chinese calligraphy simulation system, Virtual Reality Journal, 2006 (9), pp. 118-132

[17] C.L. Teo, et al. Robotic Teacher of Chinese Handwriting, Proceedings of 10th Symposium on Haptic Interfaces for Virtual Environment and Teleoperator Systems, 2002. HAPTICS 2002, 24-25, March 2002, pp. $335-341$

[18] Simbionix (Cleveland, OH, USA). http://www.simbionix.com. -2008.

[19] D. Wang, Y. Zhang, et al. "Cutting on Triangle Mesh Local Model based haptic display for dental preparation surgery simulation”, IEEE Transaction on Visualization and Computer Graphics, IEEE, 2005, pp. 671-683.

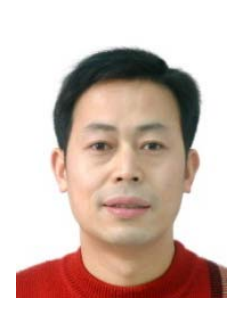

Dangxiao Wang (M'05) received the B.E. degree from Shaanxi Institute of Technology in 1997, and the M.S. degree from Northwestern Polytechnical University in 2000, and the Ph.D. degree from Beihang University, Beijing, China in 2004. Currently he is an Associate Professor at the State Key Laboratory of Virtual Reality Technology and System in Beihang University. From 2004 to 2006, he had been a post Doc at the Beihang University. After that, he was an Assistant Professor in School of Mechanical Engineering and Automation, Beihang University from 2006 to 2007.

His research interests include haptic rendering, haptic-based biometrics, and medical robotic system. He is a member of IEEE.

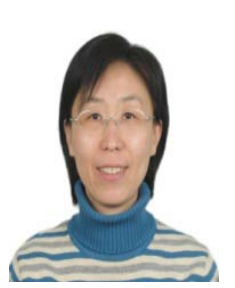

Yuru Zhang (M'95-SM'08) received the B.E. degree in mechanical engineering from Dalian University of Science and Technology, Dalian, China in 1982 and the M.S. and Ph.D. degrees in mechanical engineering from Beihang University, Beijing, China in 1984 and 1987, respectively.

Since 1987, she has been at the Beihang University where she served as the associate dean of the School of Mechanical Engineering and Automation, the associate director of Robotics Institute. Currently she is leading the Division of Human-Machine Interaction at the State Key Laboratory of Virtual Reality Technology and System. Her technical interests include haptic human-machine interface, medical robotic system, robotic dexterous manipulation, and virtual prototyping. She has published over 100 technical papers and holds 4 issued patents and 5 pending patents. She is the author of a book on robotic dexterous hands funded by the National Science Foundation of China.

Professor Zhang is a senior member of IEEE, and a member of ASME. She serves on the Board of Governors of Chinese Mechanical Engineering Society and the Advisory Board for Teaching, the Ministry of Education, China. She was awarded the Outstanding Professional for the 21 Century by the Ministry of Education and the Excellent Investigator Award by the formal Ministry of Aeronautics Industry in China.

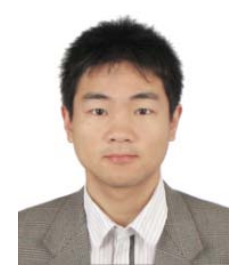

Jun Wu is a Ph.D student in the Department of Mechanical Engineering and Automation at Beihang University, Beijing, China, where he received his B.E. degree in the Department of Astronautics in 2006. He is a student member of IEEE and ASME. His research interests include physics based modeling and simulation, computer haptics, and robotics -- the computational issues therein and also their applications. 